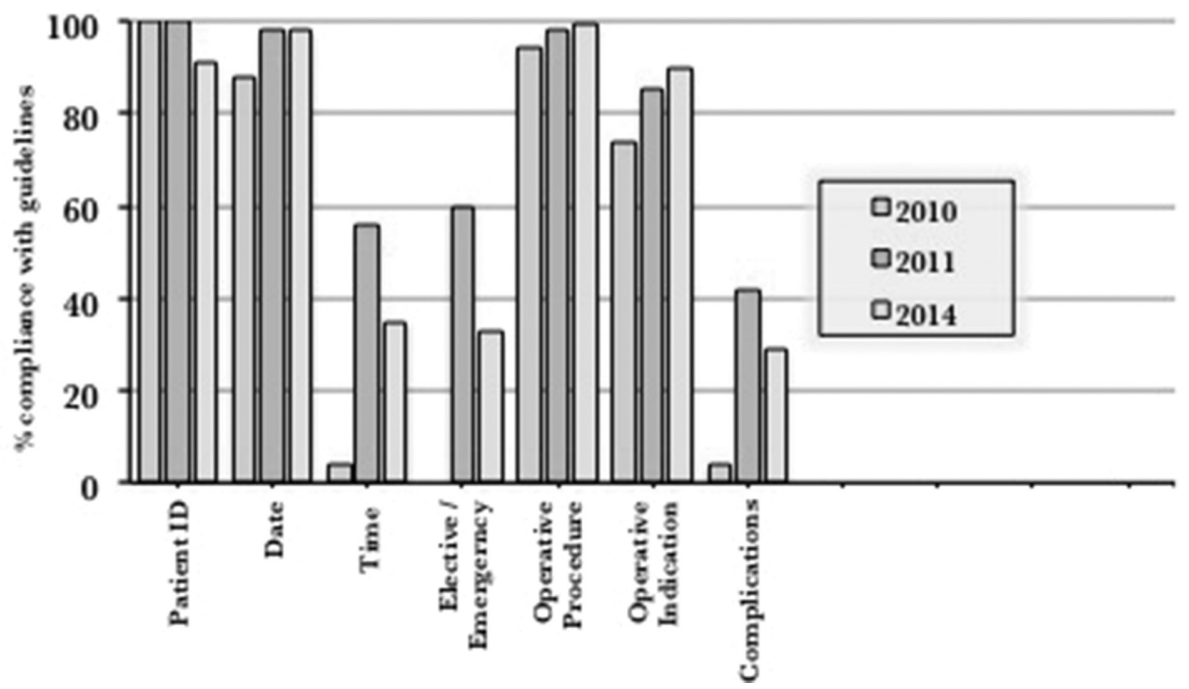

Measurement of improvement We intend to re-audit operation notes in 2-3 months' time, using an identical proforma to record data, once the proposed interventions have been implemented. We will determine whether results attained are statistically significant by calculating $\mathrm{p}$-values and using simple statistical data analysis.

Effects of changes We anticipate enhanced operative note standards as a result of introducing the proposed interventions. Our aspiration is to generate discussion in computerised documentation of surgeries and inspire greater numbers of surgeons to make use of electronic systems to ensure operative notes are both standardised and increasingly legible.

Lessons learnt Surgeons were receptive to this study's findings and were genuinely keen to improve upon their own operative documentation. Concerns were raised as to whether certain areas of documentation advised by the RCS Eng. are applicable to the paediatric populace e.g. DVT prophylaxis. This highlighted the importance of considering your patient cohort when formulating an operative note template.

Message for others We envisage the future of operative notes to be an electronic format. However, until this is achieved we recognise that the majority of surgeons will continue to use our current paper-based arrangement. Thus, we believe that typed operative documentation (using the methods described above) is an acceptable platform towards ameliorating the existing Trakcare system.

\section{G583(P) IMPROVING INTERDISCIPLINARY COMMUNICATION ON A POSTNATAL WARD}

C Duncan, E Sturdy, D Armstrong. Paediatric Department, Altnagelvin Area Hospital, Derry, UK

\subsection{6/archdischild-2015-308599.532}

Context Our hospital serves a surrounding population of over two hundred and fifty thousand people and caters to about three thousand deliveries a year. There is one postnatal ward with nineteen beds for mothers and their babies. It is usually staffed by three midwives, two healthcare assistants and one paediatric SHO during working hours.
Problem Communication between the obstetric, midwifery and paediatric staff regarding any babies on the postnatal ward was casual and ad hoc with no set verbal or written handover between daytime and out of hours staff. This appeared to lead to delays in treatment of certain babies, for instance antibiotic administration, screening for sepsis and serum bilirubin measurements.

Assessment of problem and analysis of its causes A multidisciplinary survey of those involved in obstetric and neonatal care was undertaken to identify the cause of these problems and to garner suggestions for improvement.

Intervention A more formalised handover system was developed. Information was passed between the daytime paediatric SHO to the on call team using a printed handover sheet passed from the daytime paediatric SHO to the on call team. The sheet contains a list of babies needing specific paediatric input and includes aide memoires of NICE guidelines concerning early onset neonatal sepsis and neonatal jaundice. At change of shifts patients are discussed with the written sheet as a reminder.

Study design This study was carried out as a clinical practice improvement project.

Strategy for change The printed handover sheet was introduced on a Monday morning for a trial of four weeks. It was used at each morning, early evening and night-time handover. Its introduction followed discussion with the paediatric medical staff and the midwifery team via the ward sister.

Measurement of improvement The staff of both departments were surveyed a month later to feed back any changes brought about through the new handover system. The results were very encouraging with $100 \%$ respondents reporting an improvement in communication of postnatal duties.

Effects of changes There was a improvement in both the speed at which babies were reviewed and management plans instigated. The number of bleeps to the postnatal SHO decreased. No problems were encountered throughout the period of quality improvement. The midwifery staff are now considering introducing a similar system for their daily handover.

Lessons learnt Importance of asking for feedback from all staff involved on the ward to obtain opinions on the need for change was the best way to do this. It has ensured any improvement introduced has been user friendly and staff are enthusiastic with regards to the change. 
If the authors were to repeat this quality improvement project, it would be useful to assess the implication of poor handover on infant care. For example missed/ late blood samples or delayed discharge.

Message for others The message conveyed by this project is that every ward should have an effective handover and that with using simple measures dramatic differences can be made. This project identified a lack of effective handover on a postnatal ward which led to delayed or missed patient care. Using a handover tool designed following staff feedback, handover between staff members improved significantly and this positively impacted on patient care.

Effective handover helps ensure patients receive appropriate and timely care. Potentially serious results are not forgotten about or missed when staff finish duty. It is essential as clinicians to ensure patients receive the best care and effective handover is an integral part of this.

\section{G584(P) SBAR HANDOVER TOOL: IMPROVING MATERNITY - PAEDIATRIC COMMUNICATION AT DELIVERIES}

${ }^{1}$ E Sloper, ${ }^{1} \mathrm{C}$ Edmonds, ${ }^{2} \mathrm{H}$ Bailey, ${ }^{2} \mathrm{~L}$ Lewis, ${ }^{2} \mathrm{R}$ Charlton. ${ }^{1}$ Paediatrics, Royal United Hospital Bath, Bath, UK; ${ }^{2}$ Midwifery, Royal United Hospital Bath, Bath, UK

\subsection{6/archdischild-2015-308599.533}

Context The project involves deliveries in our district general hospital delivery unit where a member of the paediatric team is requested to attend. The staff involved include midwives, neonatal nurses, paediatricians and obstetricians.

Problem Following the Root Cause Analysis (RCA) of a neonatal death at our hospital, it was recommended that improvements be made to delivery room handover between midwives and attending paediatricians. Currently handovers follow no specific pattern allowing for important information to be missed. As a result, the appropriate grade of paediatrician and correct equipment may not be present and paediatric staff may be ill-equipped to cope with the impending delivery.

Assessment of problem and analysis of its causes The RCA indicated that the condition of the baby at birth may have been predicted had the paediatrician been given a more detailed handover. A detailed handover may also have prompted them to call for senior support prior to delivery. Further discussion with paediatricians and midwives identified a lack of structure to these handovers, leaving babies at risk. Junior staff also felt that in stressful situations, prompts would ensure important information is remembered. Additionally, parents reported they would be reassured to observe a formal handover between teams.

In other high-risk transfer areas, teams complete checklists to ensure they have the necessary information, staff and equipment to minimise risk. Currently, we have no such checklist for a paediatrician entering a delivery room. Handover relies on informal discussion, often whilst staff perform other tasks. It was therefore felt that a checklist would be a useful aide in prompting a concise, complete handover.

Intervention A search was conducted to find checklists for handover between midwifery and paediatric teams in other units but none could be identified. A checklist has therefore been designed by paediatricians and approved for this study. It covers the information necessary to provide safe initial care to a baby. The checklist has been attached to every resuscitaire in the delivery unit so it is easily accessible.

Study design The checklist was introduced to midwives and paediatricians through briefings at their daily handovers. The checklist was tested on a single day and tests of change made using a Plan, Do, Study, Act (PDSA) cycle. Following further feedback from all staff groups after 2 and 4 weeks, the checklist will be amended again.

Strategy for change The change has been implemented following briefings for staff and a trial of the checklist's use. It has been amended according to initial feedback and will be attached to all resuscitaires. Further feedback will be obtained at 2 and 4 weeks after formal introduction of the checklists and they will be updated accordingly. Once formalised, the checklists will be incorporated into midwifery teaching sessions and doctor's departmental induction programmes.

Measurement of improvement Analysis will be qualitative and will focus on staff satisfaction and suggested points for improvement. Parent satisfaction will be assessed via patient surveys. Quantitative assessment of delivery outcomes cannot be undertaken within this short timescale because the number of significant neonatal resuscitations is low.

Effects of changes It is hoped that there will be improved communication in the delivery unit resulting in better anticipation and safer care, fewer crash calls for middle grade paediatricians, higher levels of staff confidence and improved parent confidence in the staff caring for their baby.

Lessons learnt We hope to learn how to devise a safe means of communicating essential information between teams.

Message for others Safe handover is a GMC requirement for good practice and we have identified a high-risk area where there are no clear guidelines for this process. Implementing our checklist has allowed the process to be formalised, making delivery room handover safer, quicker and less liable to error.

\section{G585(P) TRANSITION FROM CHILDREN'S TO ADULT DIABETES CLINIC -PATIENT'S VIEWS}

C Patankar, P Williams. Paediatrics, Medway Maritime Hospital, Gillingham, UK

10.1136/archdischild-2015-308599.534

Context The best method of providing transition of young people with diabetes is a complex and much debated subject.

Problem There is a dearth of empirical evidence on the best approach to the transition process. Involving patients is an integral part of designing and delivering this service

Intervention To obtain input from young people with diabetes regarding the current transition service in the Hospital and also invite suggestions for improvement.

Study design Young people over 16 years and adults up to age of 25 years were included. We distributed survey questionnaires via the paediatrics diabetic clinics, and also posted them to patients who were in an adult service.

Measurement of improvement Responses from the patients.

Effects of changes Results There were 200 patients between the age of 16 yrs and 25 yrs available, with 68 responses. The current system (one joint appointment with the paediatrician and the adult physician, then straight to a young adult clinic) was popular (46.3\%). Most (44.1\%) would like to stay for 1 year in the clinic but few longer than that. A significant number (71.2\%) want the adult doctor to be present in the clinic. Interestingly few (3\%) were interested in a psychologist being present. This may reflect a lack of understanding of the difficulties in the transition process by the patients. It may also reflect the lack of knowledge of how a psychologist can support them. The best time to hold the clinic seems to be at routine clinic 